\title{
Performances de ponte et qualité des œufs de poules pondeuses nourries avec des aliments à base de différentes variétés de grains de maïs
}

\author{
M.S.E. GUEDOU ${ }^{1}$, G.O. KOUATO², M.F. HOUNDONOUGBO ${ }^{2 *}$, C.A.A.M. CHRYSOSTOME² \\ et G.A. MENSAH ${ }^{1}$ \\ ${ }^{1}$ Institut National des Recherches Agricoles du Bénin (INRAB) 01 BP 884 Recette Principale, Cotonou, \\ République du Bénin. \\ ${ }^{2}$ Faculté des Sciences Agronomiques de l'Université d'Abomey-Calavi (FSA/UAC) 01 BP 526 Cotonou, \\ République Bénin. \\ *Auteur correspondant ; E-mail : fredericmh@gmail.com; Tel : (+ 229) 96142965 / 95968136
}

\section{RESUME}

L'objectif de l'étude était de comparer l'effet de quatre variétés de grains de maïs, EVDT, TZPB, AK jaune et Massawé sur les performances de ponte et la qualité des œufs des poules pondeuses de souche Hy-line. L'essai a été conduit pendant 6 mois sur des poulettes âgées de 20 semaines et réparties en 16 lots de 12 sujets chacun. Les résultats ont montré qu'il n'a pas de différence significative $(p>0,05)$ entre les variétés de grains de maïs pour le taux de ponte global à 6 mois de ponte $(62,1$ à $66,3 \%)$. A 6 mois de ponte, le poids moyen des œufs des poules pondeuses de la variété AK jaune $(55,4 \mathrm{~g})$ a été supérieur de façon hautement significative $(\mathrm{p}<0,01)$ aux poids moyens des œufs des poules pondeuses des trois autres variétés $(54,3$ à $54,5 \mathrm{~g})$. Quant aux paramètres de qualité des œufs, la couleur du jaune d'œuf des poules pondeuses nourries avec la variété AK jaune a été plus intense de façon très hautement significative $(\mathrm{p}<0,001)$ que celles des poules pondeuses alimentées avec les variétés EVDT, Massawé et TZPB. Pour tous les autres paramètres de qualité, aucune différence significative $(\mathrm{p}>0,05)$ n'a été observée entre les variétés de grains de maïs.

(C) 2018 International Formulae Group. All rights reserved

Mots clés : Maïs, taux de ponte, poids des œufs, qualité des œufs, Bénin.

\section{Spawning performance and quality of laying hen eggs fed with feeds of different varieties of maize grains}

\begin{abstract}
This study aimed to compare the effect of four maize kernel varieties, EVDT, TZPB, AK-jaune and Massawé on laying performance and egg quality in Hy-line breeding hens. The experiment was conducted during 6 months on layers of 20 weeks age and divided into 16 batches of 12 subjects each. The results showed that there was no significant difference $(\mathrm{p}>0.05)$ between the varieties of maize grains for the overall laying rate at 6 months of laying (62.1 to 66.3\%). At 6 months of lay, the average egg weight of the AK-jaune (55.4 g) layer hens was significantly ( $p<0.01$ ) higher than the average egg weights of laying hens of the three varieties (54.3 to $54.5 \mathrm{~g}$ ). As for egg quality parameters, the egg yolk color of laying hens fed with the AK-jaune variety was more intensively highly significant $(\mathrm{p}<0.001)$ than that of the laying hens fed with EVDT varieties, Massawé and TZPB. For all other quality parameters, no significant differences ( $p>0.05$ ) were observed between maize grain varieties.
\end{abstract}

(C) 2018 International Formulae Group. All rights reserved

Keywords: Maize, laying rate, egg weight, egg quality, Benin. 


\section{INTRODUCTION}

L'œuf, produit de basse-cour est une source essentielle de protéines animales. Il constitue un aliment de base dans l'alimentation humaine. Selon les estimations de la FAO, la production d'œufs de poules dans le monde a atteint 68,3 millions de tonnes en 2013, soit environ 1120 milliards d'œufs. Au Bénin, la production d'œufs de poule, est passée de 7650 tonnes (6 695368 œufs plateaux) en 2004 à 12522 tonnes (10 959398 œufs plateaux) en 2013. Les céréales sont les principales matières premières des aliments composés pour les monogastriques; elles couvrent 70 à $90 \%$ du besoin énergétique des volailles et 35 à $50 \%$ de l'apport azoté (Zitari, 2008). Parmi les céréales utilisées en aviculture, le maïs grain vient en tête. Il est donc important de chercher à exploiter au mieux cette importante matière première en aviculture. En ce sens, l'objectif de ce travail est d'évaluer l'impact de quelques variétés de maïs cultivées au Bénin sur les performances de ponte et la qualité des œufs des poules pondeuses.

\section{MATÉRIEL ET MÉTHODES Matériel animal et dispositif expérimental}

L'essai a été mené à la ferme de la Faculté des Sciences Agronomiques (FSA) de l'Université d'Abomey-Calavi (UAC) du Bénin et a porté sur 192 poules pondeuses de souche Hy-line pendant 26 semaines entre 20 et 46 semaines d'âge. D'un poids moyen initial compris entre 1100 et $1200 \mathrm{~g}$, elles ont été logées par paire dans des cages de type californien, soit au total 96 cages telles que 48 faisaient face aux vents dominants dont 24 en hauteur et 48 contre les vents dominants dont également 24 en hauteur; de façon à minimiser les effets éventuels du vent dominant et de la hauteur des cages sur les performances de ponte et la qualité des œufs. Les 192 poules ont été réparties en 16 groupes de 12 poules chacun (4 groupes par traitement). Ainsi, les 4 groupes d'un même traitement constituant ses 4 répétitions ont été logés comme suit: deux face aux vents dominants (un en bas et un en hauteur) et deux contre les vents dominants (un en bas et un en hauteur).

\section{Alimentation, abreuvement et suivi sanitaire}

Quatre (4) variétés de grains de maïs ont été comparées du point de vue performances de ponte et qualité des œufs. Il s'agit de: la variété améliorée blanche EVDT97STRW (EVDT), la variété améliorée blanche TZPBSRW (TZPB), la variété améliorée jaune AK94DMRESRY (AK jaune) et la variété locale blanche Massawé. La composition bromatologique des quatre (4) variétés de grains de maïs est présentée dans le Tableau 1.

La même formule alimentaire a été utilisée pour les 4 aliments expérimentaux issus des 4 variétés (Tableau 2). Ainsi, une variété constituait un traitement. L'aliment correspondant à chaque traitement était distribué aux 4 groupes/répétitions du traitement à raison de $115 \mathrm{~g} /$ sujet/jour durant toute la période d'essai. Les sujets ont été abreuvés ad libitum. Les mesures de prophylaxie sanitaire et de prophylaxie médicale ont été respectées pour éviter et prévenir l'apparition d'éventuelles pathologies.

\section{Collecte des données et calcul des variables}

Pour les performances de ponte, les données collectées étaient le nombre d'œufs pondus et le poids des œufs sur 6 mois; les variables calculées étaient le taux de ponte et l'indice de consommation. L'indice de consommation (IC) alimentaire a été calculé en rapportant la quantité d'aliment consommée d'une part à la masse d'œufs produite ( $g$ aliment/g œuf) et d'autre part au nombre d'œufs produits (g aliment/œuf).

Taux de ponte $=(\mathrm{N}$ œufs pondus $\mathrm{x} 100) /(\mathrm{N}$ jours ponte $\mathrm{x} N$ poules pondeuses).

Avec $\mathrm{N}$ œufs pondus = Nombre d'œufs pondus (Période considérée) ;

$\mathrm{N}$ jours de ponte $=$ Nombre de jours de ponte (Période considérée) ; 
$\mathrm{N}$ poules pondeuses $=$ Nombre de poules pondeuses (Période considérée).

Les données collectées pour l'évaluation de la qualité des œufs ont été :

- la longueur et le grand diamètre des œufs mesurés à l'aide d'une règle de pied à coulisse,

- le poids de la coquille dépourvue de la membrane interne a été pris à l'aide d'une balance électronique de sensibilité $0,1 \mathrm{~g}$,

- l'épaisseur de la coquille des œufs sans la membrane interne a été mesurée à l'aide d'un micromètre électronique de précision $0,001 \mathrm{~mm}$.

- la hauteur du blanc d'œuf (albumen) et du jaune d'œuf (vitellus) mesurée à l'aide d'un trépied micrométrique électronique de précision $0,01 \mathrm{~mm}$,

- la coloration du jaune d'œuf suivant l'éventail de Roche variant du jaune clair au jaune orange foncé dans l'ordre croissant de 1 à 15 correspondants aux différentes couleurs.

Les variables calculées pour la qualité des œufs ont été : l'Indice de Forme (IF) et l'Unité de Haugh (HU)

$\mathrm{IF}=\mathrm{GD} / \mathrm{L}$ où :
GD = Grand diamètre de l'œuf ;

$\mathrm{L}=$ Longueur de l'œuf ;

$\mathrm{HU}=100 \log \left(\mathrm{H}-1,7 \mathrm{~W}^{\mathbf{0 , 3 7}}+07,57\right)$

(Haugh, 1937) où :

$\mathrm{W}=$ Poids de l'œuf en $\mathrm{g}$;

$\mathrm{H}=$ Hauteur de l'albumen en $\mathrm{mm}$.

Par ailleurs, le coût alimentaire a été calculé en divisant le coût de l'aliment consommé par le nombre d'œufs produits dans la période considérée.

\section{Analyses statistiques}

Les données ont été analysées dans le logiciel SAS par la procédure du General Liner Model (GLM) selon le modèle suivant :

$\mathrm{Yi}=\mu+\mathrm{Ri}+\varepsilon_{\mathrm{j}}$

$\mathrm{Yi}=$ Observation des variables dépendantes

$\mu=$ Moyenne générale

$\mathrm{Ri}=$ Effet fixe de l'aliment.

$\varepsilon_{\mathrm{j}}=$ Erreur résiduelle

L'analyse de variance à un facteur a été la statistique utilisée et le test de Tukey a permis d'expliquer les moyennes significativement différentes.

Tableau 1: Composition bromatologique des quatre (4) variétés expérimentales de maïs grain

\begin{tabular}{|c|c|c|c|c|c|c|c|c|}
\hline $\begin{array}{l}\text { Variétés de } \\
\text { maïs grain }\end{array}$ & MS & CT & MO & PB & MG & CB & "EM/kg.MS & EM/kg MB \\
\hline EVDT & 89,7 & 2,7 & 97,3 & 10,9 & 4,6 & 2,1 & 3905 & 3503 \\
\hline TZPB & 89,3 & 2,8 & 97,2 & 11,3 & 4,7 & 2 & 3915 & 3496 \\
\hline AK jaune & 89,3 & 2,4 & 97,6 & 11,4 & 4,8 & 1,8 & 3955 & 3531 \\
\hline Massawé & 89,6 & 4,9 & 95,1 & 13,5 & 4,9 & 2,3 & 3814 & 3417 \\
\hline \multicolumn{9}{|c|}{$\begin{array}{l}\text { "L'Energie Métabolisable (EM) a été estimée avec l'équation de Sibbald (1980) dans Larbier et Leclercq (1994) : } \\
\text { EM vraie (en Kcal } / \mathrm{kg} . \mathrm{MS})=3951+54,4 \mathrm{MG}-88,7 \mathrm{CB}-40,8 \mathrm{CT}\end{array}$} \\
\hline \multirow{2}{*}{\multicolumn{3}{|c|}{$\begin{array}{l}\mathrm{PB}=\text { Protéines Brutes }(\text { en p100) } \\
\mathrm{CB}=\text { Cellulose Brute }(\text { en p100) }\end{array}$}} & \multicolumn{4}{|c|}{$\begin{array}{l}\mathrm{CT}=\text { Cendres Totales }(\text { en p100) } \\
\mathrm{MG}=\text { Matière Grasse }(\text { en p100) }\end{array}$} & \multicolumn{2}{|c|}{$\mathrm{MO}=$ Matière Organique $(\mathrm{en} \mathrm{p} 100)$} \\
\hline & & & $=$ Matiè & Brute & & & & \\
\hline
\end{tabular}

Tableau 2: Composition en ingrédients alimentaires et composition nutritionnelle (calculée) des aliments de poules pondeuses.

\begin{tabular}{lc}
\hline Ingrédients & Proportions \% \\
\hline Maïs & 56 \\
\hline Tourteau soja & 24 \\
\hline Tourteau coton & 8,4 \\
\hline
\end{tabular}




\begin{tabular}{|c|c|}
\hline Ingrédients & Proportions \% \\
\hline Huile de palme rouge & 1 \\
\hline Coquille d'huitre & 10 \\
\hline Lysine & 0,07 \\
\hline Méthionine & 0,15 \\
\hline Phosphate bicalcique & 0,80 \\
\hline Sel (Nacl) & 0,30 \\
\hline Prémix $^{1}(\mathrm{CMV})$ & 0,25 \\
\hline Sulfate de fer & 0,03 \\
\hline \multicolumn{2}{|l|}{ Composition nutritionnelle } \\
\hline Matière Sèche, $\%$ & 88,6 \\
\hline Cellulose brute, $\%$ & 4,16 \\
\hline Energie Métabolisable (kcal/kg MS) & 2664 \\
\hline Protéines brutes, $\%$ & 18,6 \\
\hline Lysine, \% & 0,97 \\
\hline Méthionine, $\%$ & 0,46 \\
\hline Acide aminé soufré, $\%$ & 0,78 \\
\hline Calcium, \% & 4,05 \\
\hline Phosphore total, \% & 0,57 \\
\hline
\end{tabular}

\section{RÉSULTATS}

\section{Ingestion alimentaire et indices de consommation alimentaire}

Les poules ont été rationnées; elles ont reçu et ingéré durant toute l'expérimentation $115 \mathrm{~g} / \mathrm{sujet} / \mathrm{j} . \quad$ En considérant l'indice de consommation en $\mathrm{g}$ aliment/œuf, il n'a pas été significatif $(p>0,05)$ aux mois de ponte $1,3,5$ et 6 entre les poules pondeuses ayant reçu les différentes variétés de grains de maïs (Tableau 3). Par contre, aux mois de ponte 2 et 4 , des différences significatives ont été notées $(\mathrm{p}<0,05)$. En effet, au mois de ponte 2 , l'indice de consommation (IC) des poules pondeuses ayant reçu l'aliment à base de la variété $\mathrm{AK}$-jaune a été significativement $(\mathrm{p}=$ 0,0272 ) plus élevé que l'IC des poules ayant consommé la variété TZPB. Au mois de ponte 4, l'IC des poules ayant reçu l'aliment à base de la variété $\mathrm{AK}$ jaune a été significativement $(\mathrm{p}=0,0170)$ plus faible que l'IC des poules nourries avec la variété EVDT. Sur l'ensemble des 6 mois de ponte, aucune différence significative $(p=0,2810)$, n'a été enregistrée entre les IC en $g$ aliment/œuf des poules des différents traitements qui ont oscillé entre 174,02 (TZPB) et 185,61 (Massawé).

En considérant cette fois-ci, l'IC en $\mathrm{g}$ aliment / g œuf, les mêmes observations ont été faites (Tableau 4). Toutefois, au mois de ponte 4 , l'IC des poules ayant reçu l'aliment à base de la variété $\mathrm{AK}$ jaune est significativement $(\mathrm{p}=0,0008)$ plus faible (donc meilleur) que l'IC des poules nourries respectivement avec les variétés EVDT, TZPB et Massawé.

\section{Performances de ponte \\ Taux de ponte}

Globalement, pendant les trois premiers mois de ponte, la courbe de ponte des poules ayant reçu l'aliment à base de la variété TZPB se trouvait au- dessus de celles des autres poules; elle était suivie de la courbe relative à la variété EVDT. Les trois derniers mois de ponte toutes les courbes étaient pratiquement confondues (Figure 1). Comme, nous l'avons observé au niveau des 
indices de consommation, aux mois de ponte $1,3,5$ et 6 , il n'y a pas eu de différence significative $(p>0,05)$ entre les taux de ponte des poules des différents traitements contrairement aux taux de ponte des mois 2 et 4. Au mois 2, le taux de ponte des poules de la variété TZPB $(67,26 \%)$ a été significativement $(p=0,0275)$ meilleur au taux de ponte des poules de la variété AK jaune $(57,88 \%)$. Les IC de ces poules avaient aussi évolué dans le même sens au mois de ponte 2. Au mois 4, le taux de ponte des poules de la variété AK jaune $(75,74 \%)$ a été significativement $(\mathrm{p}=0,0163)$ meilleur à celui des poules de la variété EVDT $(72,47 \%)$ à l'instar aussi de leurs indices de consommation (g aliment/œuf). Sur l'ensemble des 6 mois de ponte, il n'y a pas eu de différence significative $(p=0,2704)$ entre les taux de ponte des poules des différents traitements qui ont varié de $62,05 \%$ (Massawé) à 66,25\% (TZPB).

\section{Poids moyens des oeufs}

Pour l'ensemble des variétés de grains de maïs expérimentées, les poids moyens des œufs des poules ont diminué entre le premier et le deuxième mois de ponte, puis, ont augmenté entre le deuxième et le quatrième mois de ponte et enfin, ont diminué à nouveau les deux derniers mois (Figure 2). Au premier mois de ponte, le poids moyen des œufs des poules de la variété $\mathrm{AK}$ jaune $(60 \mathrm{~g})$ a été significativement $(\mathrm{p}=0,0002)$ supérieur aux poids moyens des œufs des poules des variétés Massawé, EVDT et TZPB (respectivement 55,1; 54,6 et 53,2 g). Aux troisième et quatrième mois de ponte, les poids moyens des œufs des poules de la variété AK-jaune (respectivement 56,5 et 56,8 g) étaient encore significativement supérieurs (respectivement $\mathrm{p}=0,0083$ et $\mathrm{p}=0,0071$ ) aux poids moyens des œufs des poules des variétés Massawé et TZPB (dans l'ordre 54,2 et $54 \mathrm{~g}$ pour le mois 3 , puis, 55,4 et $55,1 \mathrm{~g}$ pour le mois 4). Au cinquième mois de ponte, c'était plutôt le poids moyen des œufs des poules de la variété TZPB $(55,9 \mathrm{~g})$ qui était significativement $(\mathrm{p}=0,0080)$ plus élevé que les poids moyens des œufs des poules des variétés Massawé, EVDT et AK jaune (respectivement 54,3; 54,2 et 53,7 $\mathrm{g}$ ). Globalement sur les six mois de ponte, le poids moyen des œufs des poules de la variété AK jaune $(55,44 \mathrm{~g})$ a été significativement $(\mathrm{p}=0,0006)$ supérieur aux poids moyens des œufs des poules des trois autres variétés.

\section{Qualité des oufs}

Les paramètres de qualité étudiés étaient : l'indice de forme, la couleur du jaune d'œuf, la hauteur du jaune d'œuf, la longueur du jaune d'œuf, la hauteur du blanc d'œuf, la longueur du blanc d'œuf, le poids de la coquille d'œuf, l'épaisseur de la coquille d'œuf et l'unité de Haugh (Tableau 4). Au nombre de ces paramètres, seule la couleur du jaune d'œuf a affiché une différence significative par rapport aux poules ayant reçu les aliments issus des différentes variétés. En effet, comme c'était prévisible, la couleur du jaune d'œuf des poules nourries avec la variété $\mathrm{AK}$ jaune a été significativement $(\mathrm{P}<0,0001)$ plus intense que celles des poules alimentées respectivement avec les variétés EVDT, Massawé et TZPB (Tableau 5).

\section{Coûts alimentaires}

Indépendamment des variétés, les coûts alimentaires (FCFA/œuf) du premier mois de ponte ont été supérieurs à ceux des cinq autres mois qui ont suivi (Figure 3 ). Cette situation s'explique par les valeurs élevées des indices de consommation alimentaire (g aliment / œuf) au premier mois de ponte. En effet, au début de ponte, les poules n'ont pas une bonne conversion alimentaire en œufs, le taux de ponte est faible. Pour toutes les quatre variétés de grains de maïs testées, les courbes de coûts alimentaires des poules pondeuses ont été similaires sur les cinq derniers mois de ponte. Toutefois, d'une part, le coût alimentaire du mois deux des poules de la variété $\mathrm{AK}$ jaune a été significativement ( $\mathrm{p}=0,0272$ ) plus élevé que celui des poules de la variété TZPB. (49,9 contre 42,8 FCFA/œuf). Et d'autre part, le coût alimentaire du mois quatre des poules de la 
variété $\mathrm{AK}$ jaune a été significativement $(0,0168)$ plus faible que celui des poules de la variété EVDT (37,9 contre 39,7 F CFA/œuf). En effet, les valeurs des coûts alimentaires sont proportionnelles à celles des indices de consommation avec lesquelles, nous avons eu les mêmes tendances par rapport aux variétés de grains de maïs. Sur l'ensemble des six mois de ponte, il n'y a pas eu de différence significative $(p=0,2807)$ entre les coûts alimentaires des poules nourries avec les différentes variétés.

Tableau 3: Indices de consommation en $g$ aliment/œuf des poules pondeuses nourries avec des aliments à base de différentes variétés de grains de maïs.

\begin{tabular}{llllll}
\hline & EVDT & AK jaune & Massawé & TZPB & Prob \\
\hline IC 1 & 369,10 & 267,39 & 440,90 & 271,03 & 0,2038 \\
$\mathrm{IC}_{2}$ & $173,85^{\mathrm{ab}}$ & $199,67^{\mathrm{a}}$ & $183,77^{\mathrm{ab}}$ & $171,16^{\mathrm{b}}$ & 0,0272 \\
$\mathrm{IC}_{3}$ & 157,05 & 160,66 & 155,89 & 157,00 & 0,6852 \\
$\mathrm{IC}_{4}$ & $158,73^{\mathrm{a}}$ & $151,85^{\mathrm{b}}$ & $156,30^{\mathrm{ab}}$ & $156,79^{\mathrm{ab}}$ & 0,0170 \\
$\mathrm{IC}_{5}$ & 162,54 & 163,85 & 163,03 & 161,41 & 0,8933 \\
$\mathrm{IC}_{6}$ & 178,79 & 180,11 & 184,51 & 181,25 & 0,7083 \\
$\mathrm{IC}_{\mathrm{G}}$ & 180,80 & 180,46 & 185,61 & 174,02 & 0,2810 \\
\hline
\end{tabular}

Les moyennes ne partageant aucune lettre en commun sont significativement différentes

$\mathrm{ICn}=$ Indice de consommation du $\mathrm{n}^{\text {ième }}$ mois de ponte

ICG = Indice de consommation global

Prob $=$ Probabilité

Tableau 4: Indices de consommation en $g$ aliment/ $g$ œuf des poules pondeuses nourries avec des aliments à base de différentes variétés de grains de maïs.

\begin{tabular}{llllll}
\hline & EVDT & AK jaune & Massawé & TZPB & Prob \\
\hline $\mathrm{IC}_{1}$ & 6,78 & 4,46 & 8,08 & 5,09 & 0,1946 \\
$\mathrm{IC}_{2}$ & $3,34^{\mathrm{ab}}$ & $3,74^{\mathrm{a}}$ & $3,485^{\mathrm{ab}}$ & $3,24^{\mathrm{b}}$ & 0,0458 \\
$\mathrm{IC}_{3}$ & $2,82 \mathrm{ab}$ & 2,84 & 2,88 & 2,91 & 0,7599 \\
$\mathrm{IC}_{4}$ & $2,83^{\mathrm{a}}$ & $2,67^{\mathrm{b}}$ & $2,82^{\mathrm{a}}$ & $2,85^{\mathrm{a}}$ & 0,0008 \\
$\mathrm{IC}_{5}$ & 3,00 & 3,05 & 3,00 & 2,89 & 0,2165 \\
$\mathrm{IC}_{6}$ & 3,29 & 3,38 & 3,42 & 3,33 & 0,6058 \\
$\mathrm{ICG}$ & 3,31 & 3,25 & 3,42 & 3,20 & 0,2224 \\
\hline
\end{tabular}

Les moyennes ne partageant aucune lettre en commun sont significativement différentes

$\mathrm{ICn}=$ Indice de consommation du $\mathrm{n}^{\text {ième }}$ mois de ponte;

$\mathrm{ICG}=$ Indice de consommation global

Prob $=$ Probabilité 


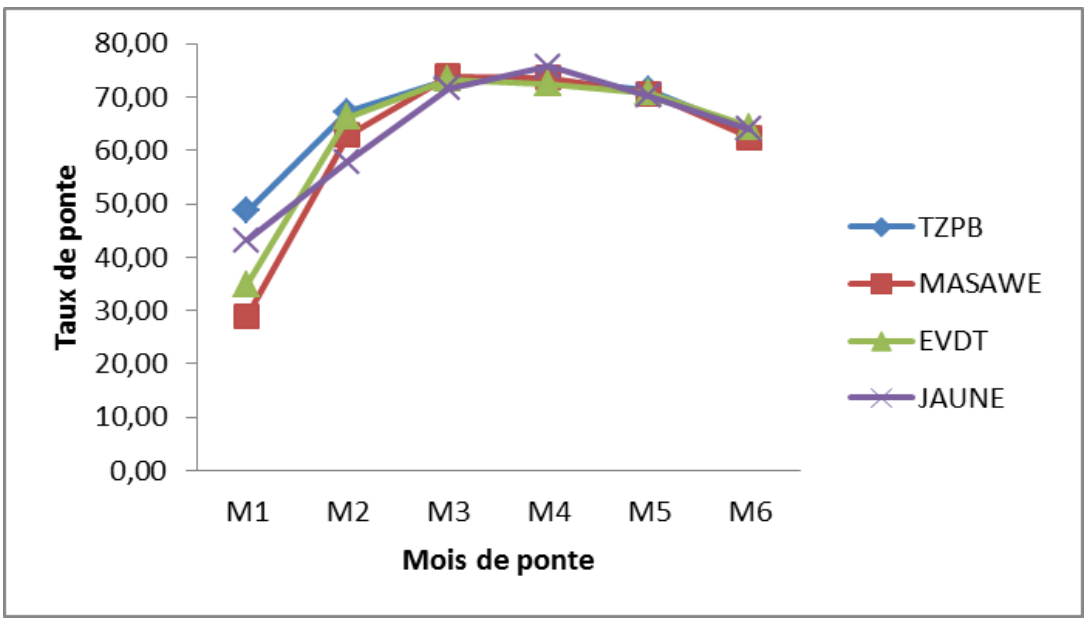

Figure 1: Evolution du taux de ponte en fonction des mois de ponte de poules pondeuses nourries avec des aliments à base de différentes variétés de grains de maïs.

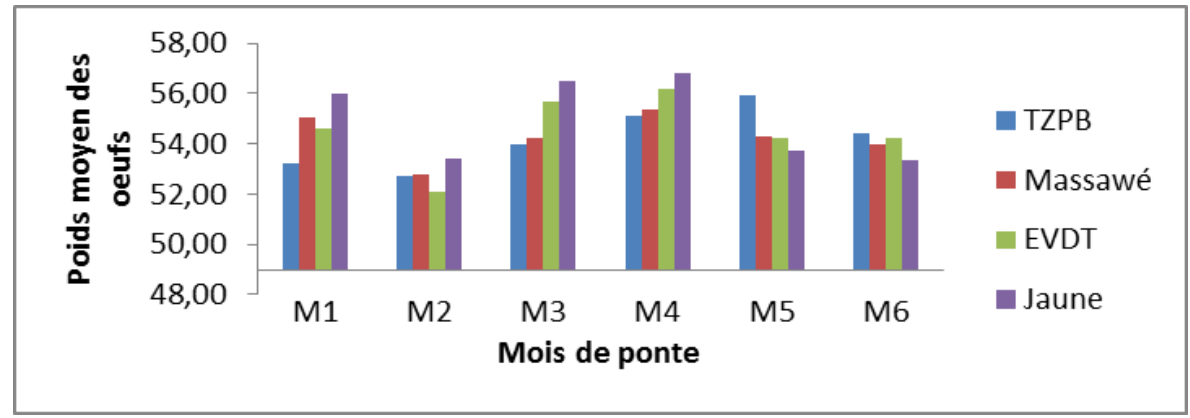

Figure 2 : Evolution du poids moyen des œufs en fonction des mois de ponte des poules nourries avec des aliments à base de différentes variétés de grains de maïs.

Tableau 5: Qualités externe et interne des œufs des poules pondeuses nourries avec des aliments à base de différentes variétés de maïs.

\begin{tabular}{llllllllll}
\hline Variétés & \multicolumn{9}{l}{ Qualités des œufs } \\
\cline { 2 - 9 } & IF & $\mathbf{C ~ J}$ & $\mathbf{H ~ J}$ & $\mathbf{L ~ J}$ & $\mathbf{H ~ B ~}$ & $\mathbf{L B}$ & UH & P cq & É cq \\
EVDT & 0,81 & $3,94^{\mathrm{a}}$ & 18,57 & 4,04 & 9,43 & 7,51 & 95,94 & 8,69 & 0,40 \\
AK jaune & 0,81 & $7,63^{\mathrm{b}}$ & 18,16 & 3,96 & 9,19 & 7,57 & 94,47 & 8,63 & 0,36 \\
Massawé & 0,78 & $4,31^{\mathrm{a}}$ & 18,00 & 4,04 & 9,60 & 7,64 & 96,38 & 8,31 & 0,36 \\
TZPB & 0,80 & $3,19^{\mathrm{a}}$ & 17,57 & 3,99 & 9,33 & 7,39 & 95,84 & 8,81 & 0,40 \\
ES & 0,04 & 1,28 & 1,38 & 0,21 & 1,49 & 0,63 & 7,48 & 1,19 & 0,05 \\
Probabilité & 0,054 & $<0,0001$ & 0,240 & 0,703 & 0,890 & 0,698 & 0,898 & 0,676 & 0,039 \\
\hline
\end{tabular}

\footnotetext{
Les moyennes suivies de lettres différentes dans la même colonne sont significativement différentes

$\mathrm{IF}=$ Indice de forme $\quad \mathrm{C} \mathrm{J}=$ Couleur du jaune $\quad \mathrm{H} \mathrm{J}=$ Hauteur du jaune

$\mathrm{LJ}=$ Longueur du jaune $\quad \mathrm{HB}=$ Hauteur du blanc $\quad \mathrm{LB}=$ Longueur du blanc

$\mathrm{UH}=$ Unité Haugh,$\quad \mathrm{P} c q=$ Poids coquille $\quad \mathrm{E} c \mathrm{c}=$ Epaisseur coquille

Longueurs, hauteurs et épaisseurs en mmm et poids en $\mathrm{g}$.
} 


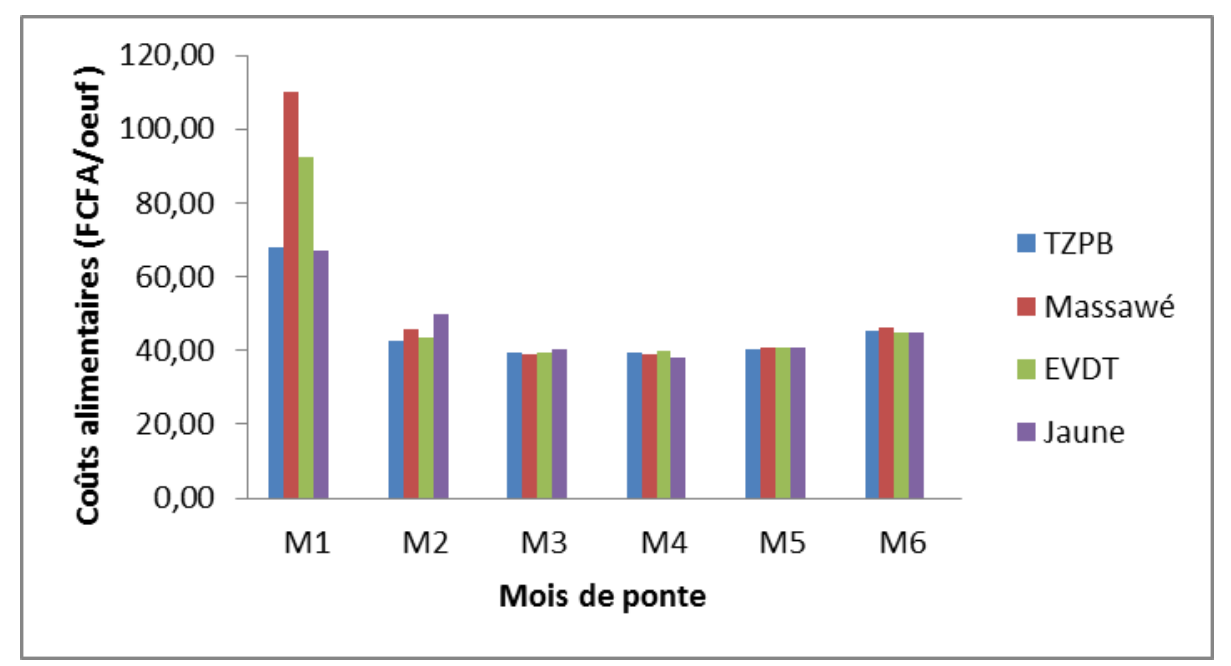

Figure 3: Evolution du coût alimentaire en fonction des mois de ponte des poules nourries avec des aliments à base de différentes variétés de grains de maïs.

\section{DISCUSSION \\ Indices de consommation}

Les indices de consommation en $\mathrm{g}$ aliment /œuf, que nous avons obtenus chez les poules Hy-line au premier mois de ponte $(267,39$ à 440,90) sont comparables à ceux relatés (239 à 463) par Houndonougbo et al. (2012) sur des poules pondeuses ISA Brown alimentées avec des rations à base de feuilles séchées de manioc. Il en a été quasiment de même, le troisième mois de ponte (157 à 160,66) pour notre essai contre les 134 à 165 obtenu par (Houndonougbo et al., 2012). Par contre au deuxième mois de ponte, les IC en $\mathrm{g}$ aliment/ œuf, que nous avons enregistrés $(171,16$ à 199,7) sont moins intéressants que ceux trouvés (134 à 159) par les mêmes auteurs. Les indices de consommation en $\mathrm{g}$ aliment/g œuf que nous avons enregistrés le troisième mois de ponte, soit 13 semaines de ponte $(3,20$ à 3,42$)$ ont été pratiquement le double des IC $(1,3$ à 1,6$)$ auxquels ont abouti Diomandé et al. (2008) sur des poules ISA Brown à 14 semaines de ponte dans une étude de substitution de la farine de poisson par celle d'escargot. Aussi, les IC en g aliment/g œuf de nos poules pondeuses ont été supérieurs aux 2,74 à 2,98 de Bourin et Bouvarel (2015) sur des poules pondeuses entre 30 et 36 semaines d'âge.

\section{Performances de production des œufs}

Les taux de ponte obtenus aux premier, deuxième et troisième mois de ponte (respectivement 29,0 à 48,7\%;57,89 à 67,3\% et 71,6 à $73,8 \%$ ) ont été moins intéressants que ceux trouvés (respectivement 42,9 à $53,7 \%$; 71,7 à $84,7 \%$ et 71,7 à $81,3 \%$ ) par Houndonougbo et al. (2012). Les taux de ponte globaux de nos poules à 6 mois de ponte, entre 20 et 46 semaines d'âge (62,0 à $66,2 \%)$ sont aussi en deçà de ceux signalés (68,8 à 74,9\%) par Tossou et al. (2014) entre 26 et 41 semaines d'âge. Les écarts sont encore plus élevés lorsqu'on les compare aux taux de ponte trouvés par Bourin et Bouvarel (2015) entre 30 et 36 semaines d'âge, soit 7 à 8 mois un- demi environ de ponte $(91,3$ à $92,0 \%)$. En revanche, les taux de ponte obtenus dans cette étude à trois mois de ponte, soit 13 semaines $(71,6$ à $73,8 \%)$ sont nettement meilleurs à ceux évoqués par Diomandé et al. (2008) à 14 semaines de ponte $(51,9$ à $65,4 \%)$. Ces différents taux de ponte s'expliquent essentiellement par la souche de poules pondeuses, la période de ponte considérée et la quantité de protéines et d'acides aminés ingérée quotidiennement par les poules.

Les poids moyens d'œufs obtenus par mois, toutes variétés de grains de maïs 
confondues ont varié entre $53 \mathrm{~g}$ et $60 \mathrm{~g}$. D'après la catégorisation des œufs suivant le calibre, effectuée par Mantsanga et al. (2016), cet intervalle de poids correspond aux œufs de calibre standard (œufs ayant un poids moyen). Ces 53 à $60 \mathrm{~g}$ de poids moyens d'œufs concordent avec ceux obtenus par Houndonougbo et al. (2012) et qui varient entre un peu moins de $50 \mathrm{~g}$ et $60 \mathrm{~g}$ environ. Comparés aux résultats $(61,8$ à $60,4 \mathrm{~g})$ de Bourin et Bouvarel (2015), les œufs de nos poules ont été moins lourds; par contre, ils ont été meilleurs aux 57,5 g enregistrés par Diomandé et al. (2008). Tout comme le taux de ponte, le poids des œufs est fortement contrôlé par la quantité de protéines et d'acides aminés ingérée quotidiennement par les poules. Environ 75 à $80 \%$ des acides aminés assimilés par une poule sont directement utilisés pour la production de l'œuf (ISA, 2010). L'ingestion d'un gramme de protéines par jour en plus entraîne en moyenne une augmentation du poids de l'œuf de 1,3 g dans le cas de protéines équilibrées en acides aminés (Bouvarel et al., 2010). Les acides aminés qui présentent le plus fort effet sur le poids de l'œuf lorsqu'ils sont limitants sont tout d'abord la méthionine, puis la thréonine, la valine et enfin la lysine (Bregendahl et al., 2008).

\section{Qualités des œufs}

Les valeurs trouvées pour l'indice de forme (IF) des œufs de poules pondeuses, et pour l'épaisseur de la coquille dans ce travail sont comparables à celles signalées (respectivement 0,70 à 0,80 et 0,3 à $0,4 \mathrm{~mm}$ ) par Diomandé et al. (2008). Les valeurs obtenues par Houndonougbo et al. (2012) pour les paramètres de qualité tels que l'indice de forme $(0,78)$, la hauteur du jaune d'œuf $(17,5$ à $18,1 \mathrm{~mm})$, l'épaisseur de la coquille $(0,37$ à $0,39 \mathrm{~mm})$ sont similaires à nos résultats. Quant aux paramètres comme la hauteur du blanc $(7,57$ à $8,20 \mathrm{~mm})$, l'Unité Haugh $(85,1$ à 90,5$)$ et le poids de la coquille (7,52 à 7,66 g), les valeurs obtenues par ces auteurs sont inférieures à celles que nous avons enregistrées. Par ailleurs, la couleur du jaune d'œuf issu de l'aliment à base de la variété AK jaune a été plus intense que celle provenant des aliments utilisés par Houndonougbo et al. (2012) où il n'y a pas eu de maïs jaune. Dans une étude d'alimentation $100 \%$ bio pour les poules pondeuses effectuée par Bourin et Bouvarel (2015) sur la féverole comme une éventuelle alternative au soja, l'Unité Haugh $(83,59$ à 90,97) et le poids de la coquille $(5,84$ à $6,40 \mathrm{~g})$ relatés sont nettement en deçà de nos résultats.

\section{Coûts alimentaires des oufs}

Les coûts alimentaires obtenus sur les six mois de ponte (43 à 46 FCFA/œuf) sont inférieurs au 52 FCFA/œuf mais supérieurs au 39 FCFA/œuf trouvés par Mantsnga et al. (2016) respectivement pour une alimentation complète et une alimentation calcique séparée chez les poules pondeuses. Les coûts alimentaires de nos poules, pour chacun des trois premiers mois (dans l'ordre 67 à 110 FCFA/œuf, 43 à 50 FCFA/œuf et 39 à 40 FCFA/œuf) sont tous plus élevés que ceux signalés par Houndonougbo et al. (2012) dans une étude de rations alimentaires à base de feuilles séchées de manioc (respectivement 49 à $90 \mathrm{FCFA} /$ œuf, 28 à $30 \mathrm{FCFA} / œ u f$ et 28 à 32 FCFA/œuf). Ceci s'explique par le coût au kilogramme de provende qui a varié de 187 FCFA à 207 FCFA dans le cas de l'étude sur les feuilles de manioc alors qu'il a été de 250 FCFA pour ce qui nous concerne. En effet, le second facteur qui explique la variation des coûts alimentaires, à savoir l'indice de consommation, a été globalement similaire pour les deux études.

\section{Conclusion}

Les poules pondeuses ayant reçu l'aliment à base de la variété blanche améliorée TZPB ont donné le meilleur taux de ponte qui du point de vue statistique est semblable aux taux de ponte des poules pondeuses des trois autres traitements (variété améliorée jaune AK jaune, variété améliorée blanche EVDT et variété locale blanche Massawé). La variété AK jaune a engendré le meilleur poids moyen d'œufs de façon significative comparativement aux poids moyens d'œufs induits par les trois autres variétés. Quant aux paramètres de la qualité des œufs, (indice de forme, Unité de Haugh, la 
couleur du jaune d'œuf, poids de la coquille, épaisseur de la coquille, etc.), ils sont similaires chez les poules pondeuses des quatre variétés à l'exception de la couleur du jaune d'œuf qui est plus intense chez les poules nourries avec le maïs $\mathrm{AK}$-jaune.

\section{CONFLITS D'INTERETS}

Les auteurs déclarent n'avoir aucun conflit d'intérêts.

\section{CONTRIBUTIONS DES AUTEURS}

MSEG a bénéficié de la bourse du PPAAO pour ses travaux en vue de l'obtention du Diplôme Doctorat; GOK a participé à la mise en forme suivant les exigences de la revue; MFH a participé à l'amélioration de la qualité scientifique de ce manuscrit; Le dispositif expérimental a été mis en place par le laboratoire de CAAMC sans oublier son apport technique et scientifique. GAM a beaucoup apporté sa contribution scientifique à l'amélioration de la qualité de cet article.

\section{REMERCIEMENTS}

Nos remerciements vont, particulièrement à l'endroit du Fond National de la Recherche Scientifique et Technique (FNRSIT) qui a travers le Projet Ressources Alimentaires pour Animaux (RAPA) à contribuer à la publication de ce manuscrit. Nous ne saurions oublier l'appui technique du Laboratoire de Recherche Avicole et de ZooEconomie (LaRAZE).

\section{REFERENCES}

Bourin M, Bouvarel I. 2015. Alimentation $100 \%$ bio pour les poules pondeuses : Résultats d'essais conduits en station expérimentale. Vers une alimentation $100 \% \mathrm{AB}$ en élevage avicole biologique - Restitution des programmes ICOPP, Proté AB, AviAlim Bio, Avibio, Monalim Bio, Angers ITAB/CRAPDL/IBB/ITAVI/INRA, 9 p.
Bouvarel I, Nys Y, Panhéleux M, Lescoat P. 2010. INRA Productions Animales, 23(2) : 167-182.

Bregendahl K, Roberts SA, Kerr B, Hoehler D. 2008. Poultry Science, 87 : 744- 758.

Diomandé M, Kippré VA, Koussémon M, Kaménan A. 2008. Substitution de la farine de poisson par celle d'escargot (Achatina fulica) dans l'alimentation des poules pondeuses en Côte d'Ivoire. Livestock Research for Rural Development, 20(1): $12 \mathrm{p}$.

Houndonougbo MF, Chrysostome CAAM, Houndonougbo VP. 2012. Performances de ponte et qualité des œufs des poules pondeuses ISA Brown alimentées avec des rations à base de feuilles séchées de manioc (Manihot esculenta, Crantz). Int. J. Biol. Chem. Sci., 6(5): 1950-1959.

ISA. A Hendrix Genetics Company. 2010. Conduite technique des élevages de poulettes et de pondeuses en climat chaud. $17 \mathrm{p}$.

Mantsanga HB, Amona I, Banga-Mboko H, Bakana MA, Adzona PP. 2016. Effet de l'alimentation calcique séparée sur la qualité de la coquille, le calibre, et le cout de production de l'œuf chez la poule Lhoman Brown en zone tropicale. Journal of Applied Biosciences, 97: 9212-9219.

Tossou ML, Chrysostome CAAM, Houndonougbo MF, Missohou A, Abiola FA. 2014. Influence du système de logement sur quelques performances zootechniques et économiques des poules pondeuses au Sud-Bénin. Agronomie Africaine, 26(2) : 147 - 154.

Zitari S. 2008. Etude des valeurs nutritives de certaines ressources alimentaires locales utilisées dans l'alimentation des animaux. Master, Université de Sousse, $52 \mathrm{p}$. 